\title{
Light Polarization Using Ferrofluids and Magnetic Fields
}

\author{
Alberto Tufaile, ${ }^{1}$ Timm A. Vanderelli, ${ }^{2}$ and Adriana Pedrosa Biscaia Tufaile ${ }^{1}$ \\ ${ }^{1}$ Soft Matter Laboratory, Escola de Artes, Ciências e Humanidades, Universidade de São Paulo, 03828-000 São Paulo, SP, Brazil \\ ${ }^{2}$ Ferrocell USA, 739 Route 259, Ligonier, PA 15658, USA \\ Correspondence should be addressed to Alberto Tufaile; tufaile@usp.br
}

Received 5 December 2016; Accepted 23 March 2017; Published 9 May 2017

Academic Editor: Charles Rosenblatt

Copyright (C) 2017 Alberto Tufaile et al. This is an open access article distributed under the Creative Commons Attribution License, which permits unrestricted use, distribution, and reproduction in any medium, provided the original work is properly cited.

We are presenting an experimental setup based on polarized light, enabling the visualization of the magnetic field of magnetic assemblies using a Hele-Shaw cell filled with ferrofluids. We have simulated the observed patterns with hypergeometric polynomials.

\section{Introduction}

When a strong magnet is brought close to a Hele-Shaw cell containing a ferrofluid, this magnetic fluid undergoes structural transitions due to the magnetic field, forming linear chains or rods of nanoparticles along the field direction. This is a magnetooptic effect in which an electromagnetic wave propagates through the ferrofluid that has been altered by the presence of a quasi-static magnetic field, forming some patterns. One way to observe this pattern formation is to use polarized light [1-4].

Some interesting applications of this type of device include optical switches, or the simulation of atmospheric optics known as jumping laser dogs [5], just to cite few of them. Another potential application is characterizing and controlling the quality of compound magnets observing the patterns formed in this type of Hele-Shaw cell filled with ferrofluids, known as Ferrocell.

In this paper, we present how to use and characterize the magnetic field of compound magnets using the Ferrocell, analyzing some aspects of the pattern formation and their relationship with some optical properties obtained from the presence of different distribution of the magnetic field, obtaining a map of the magnetic field.

\section{Materials and Methods}

The diagram of the experiment is shown in Figure 1(a). The Ferrocell is placed between two crossed polarizers illuminated with white light. The white light source was generated using a LED panel light $(12 \mathrm{~W})$, illuminating over the complete surface of a square Ferrocell $(22 \mathrm{~mm} \times$ $22 \mathrm{~mm}$ ) homogeneously, with a methacrylate diffuser and backlighting technique. The glass plates used in the Ferrocell in this case are microscope slides. The image is obtained directly from the system polaroids/Ferrocell, photographing the Ferrocell with a digital camera.

The ferrofluid is the EFH1, a stable colloidal dispersion using light mineral oil. The response time is of the order of $200 \mathrm{~ms}$, and any pattern disappears almost instantly after removing the magnetic field. In very low fields, the ferrofluid is isotropic, and the polarized light passes through the Ferrocell. When the magnetic field is increased, we have the formation of needlelike structures of the ferrofluid. These structures are shown in Figure 1(b), using an optical microscope (200x magnification). These structures are formed by the ferrofluid in the presence of the magnetic field, ranging from $100 \mathrm{G}$ to $500 \mathrm{G}$, creating some elongated structures, known as needlelike ferrofluid structures.

The magnets are placed at the top of the analyzer, as it is shown in Figure 1(a), and they can create different magnetic field configurations, depending on their orientation, measuring the magnetic field intensity with a Hall sensor, as it is shown in Figure 2(a). For example, a cylindrical magnet with the north pole facing the center system formed by the analyzer/Ferrocell/polarizer in vertical position can create the unipolar field configuration shown in the first plot of Figure 2(b). Another possibility is to place the same magnet in the horizontal position with the north pole and south pole in direct contact with the analyzer/Ferrocell/polarizer 


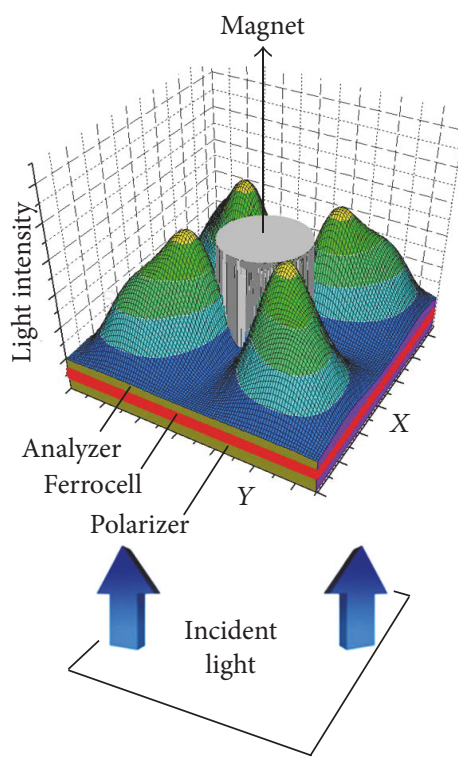

(a)

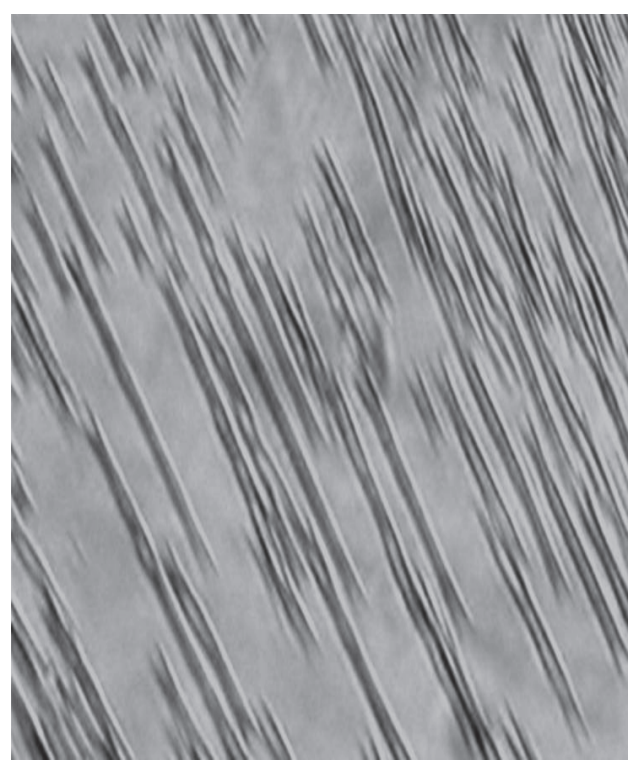

(b)

FIGURE 1: Experimental apparatus in (a), with the surface plot of the light intensity. Optical microscopic view (200x) of the structures formed by a ferrofluid in the presence of a magnetic field (from $100 \mathrm{G}$ to $500 \mathrm{G}$ ) creating some needlelike structures in (b).

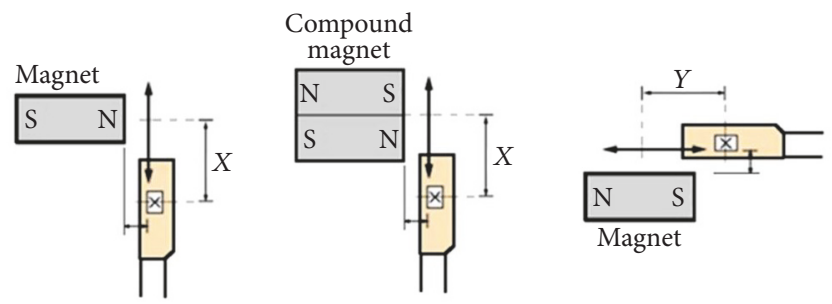

(a)

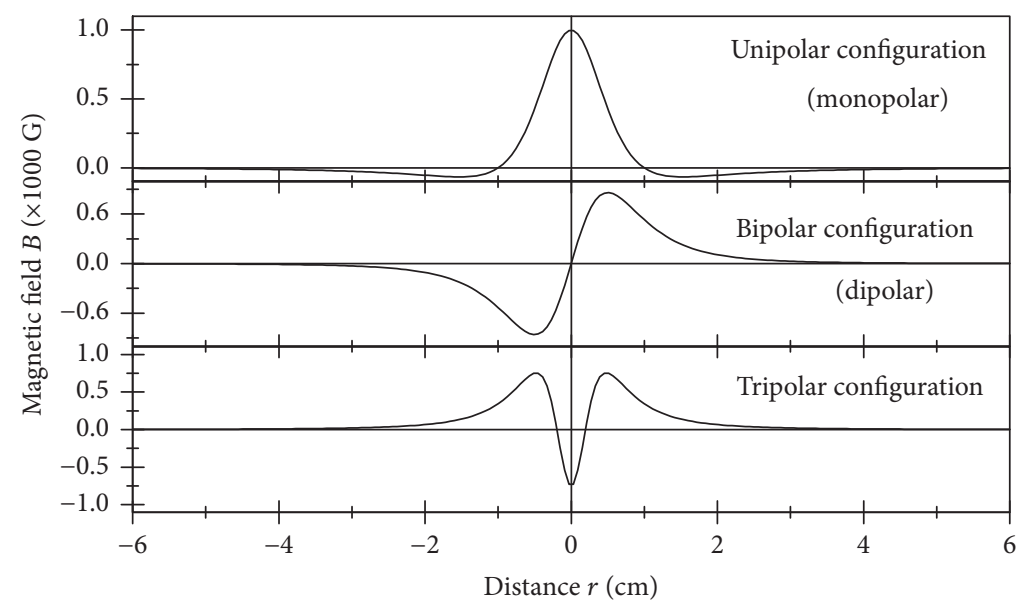

(b)

FIGURE 2: (a) The diagram of the measurements using the Hall sensor for the magnetic field of the compound magnets, in order to carry out a comparison with the light patterns observed in the Ferrocell. In (b), there are plots of the magnetic fields for different compound magnets. The coordinate $r$ represents a generic coordinate, which can be replaced by $x$ and $y$, or it represents the polar coordinate, depending on the magnetic field configuration. 


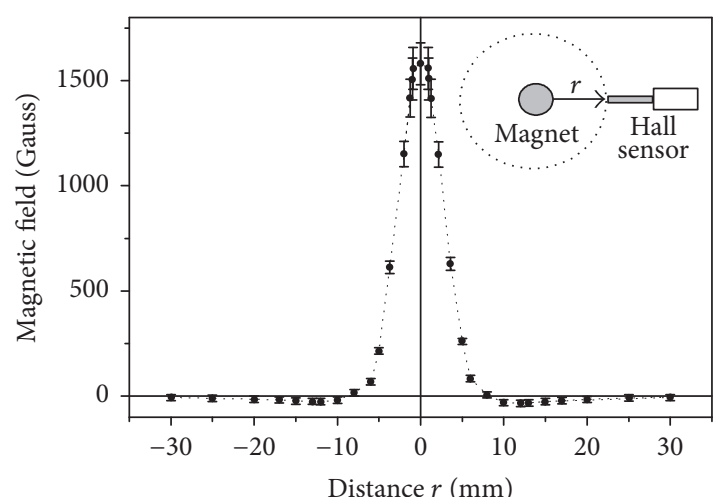

(a)

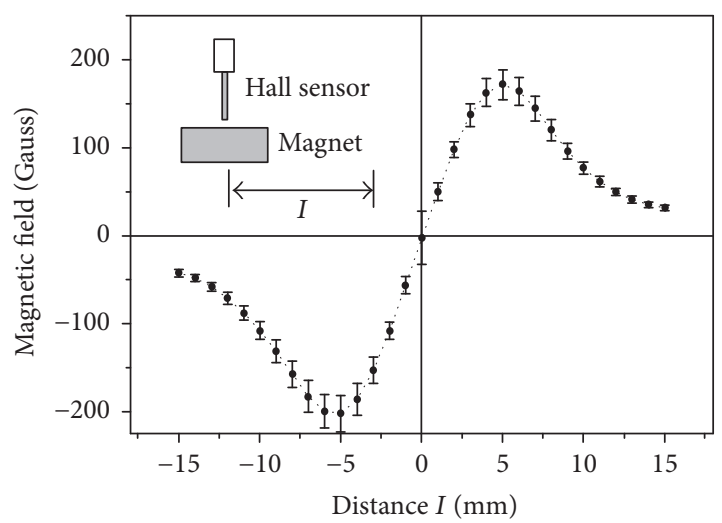

(c)

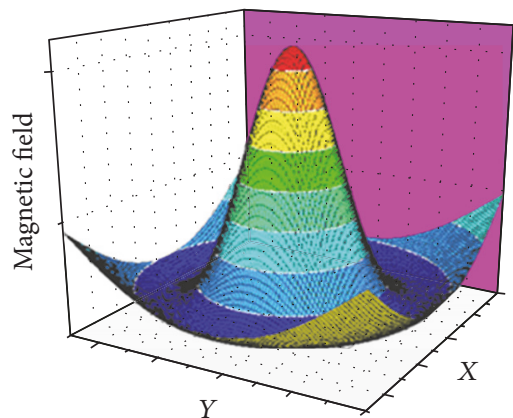

(b)

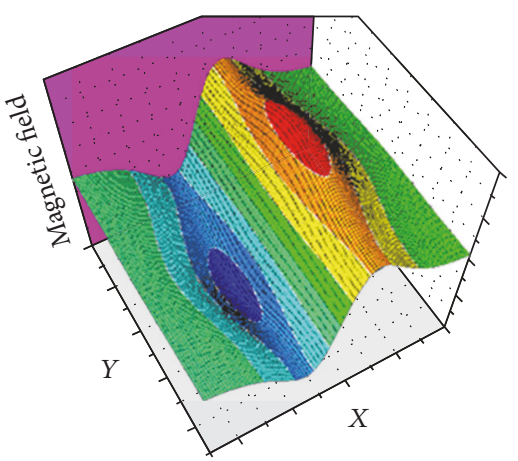

(d)

Figure 3: Plots representing the magnetic field using the Gaussmeter. (a) The plot of the magnetic field in the monopolar configuration (also known as unipolar configuration) and (b) the two-dimensional plot of the intensity of the magnetic field. (c) The plot of the magnetic field in the bipolar configuration and (d) the two-dimensional plot of the intensity of the magnetic field. When the magnet is placed at the top of the Ferrocell, the ferrofluid reacts to one of these configurations depending on its position.

system, creating the magnet field configuration of bipolar configuration of the second plot of Figure 2(b). Because the Hall sensor just measures the magnetic field at one point in the space at time, in order to reconstruct the magnetic field intensity in a similar plane of the Ferrocell, we have to slide it in certain directions. For example, the unipolar configuration of the diagram of the Hall sensor in Figure 2(a) and in the magnetic field intensity of Figure 2(b) is represented in Figure 3(a) with polar coordinates and with Cartesian coordinates in Figure 3(b). Placing the same magnet in the horizontal position at the center of the Ferrocell, the magnetic field in the plane of the Ferrocell of the bipolar configuration of Figure 2(b) is equivalent to the magnetic field represented in Figures 3(c) and 3(d).

We have used neodymium (Ne-Fe-B) magnets facing the center of the Ferrocell in order to change the light patterns. For example, the values of the magnetic field of some compound magnets were obtained with a Lakeshore Gaussmeter model 475 DSP in the plots of Figure 3, at room temperature of $27^{\circ} \mathrm{C}$, with a Hall sensor. We can reconstruct the intensity of the magnetic field around the magnets using these data, as it is shown in Figure 3. The nanostructures inside the Ferrocell align to these local configurations of the magnetic field, whenever the magnet is placed at the top of the Ferrocell. In this way, the monopolar configuration is one of the magnetic poles facing directly the Ferrocell, and it is almost the half part of the bipolar configuration of Figure 3(d).

In order to simulate the polarized light patterns observed in the Ferrocell, we have used some routines written in commercial software (OriginLab), computing matrices with $1000 \times 1000$ elements.

\section{Results and Discussion}

The distinguishing basic feature of the Ferrocell in the presence of magnetic field, as far as optical properties are concerned, is the fact that the Ferrocell is generally magnetically anisotropic, such as the case of the Cotton-Mouton effect [5], in which the substance inside the Ferrocell behaves like a large group of small compasses, with the magnetic field defining the optic orientation. This means that the polarization in the Ferrocell by a given magnetic field is not just a simple scalar constant times the field but varies in a manner that depends on the direction of the applied field in relation to the plane of the Hele-Shaw cell. In this way, the polarization is attributed to the alignment of the nanoparticles by the magnetic field. In Figure 4, we present the light pattern obtained with a 


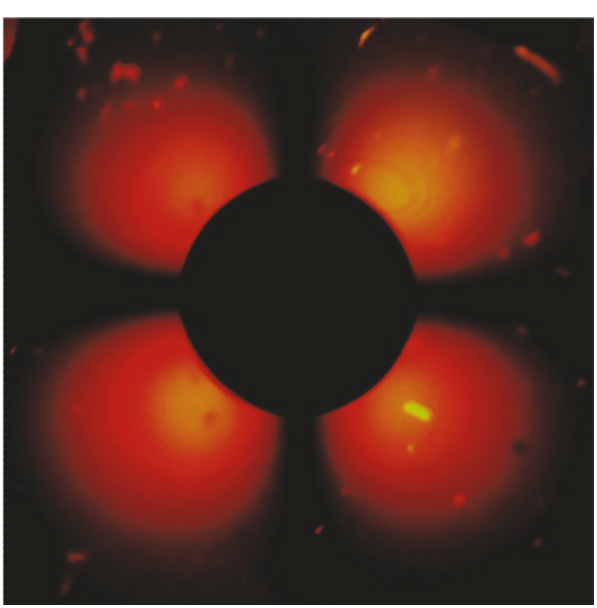

(a)

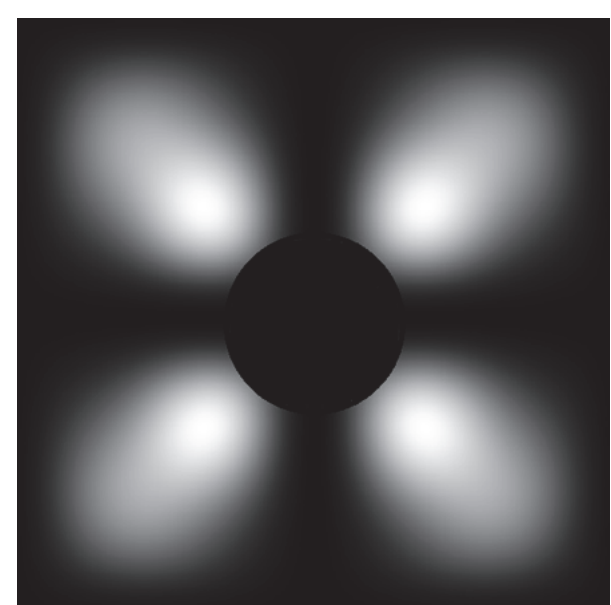

(b)

Figure 4: (a) The pattern of light polarization from the Ferrocell with a cylindrical magnet placed in the monopolar configuration. The simulation of this configuration is presented in (b).

cylindrical magnet placed at the Ferrocell in the monopolar (or unipolar) configuration. This light pattern is associated with the magnetic field of Figure 3(b).

Using the information obtained from this pattern, we can explain the observed patterns with a phenomenological model. In order to obtain this model, we need to know the mathematical expression of the external magnetic field and the equation representing the needlelike structure. First, the calculation of the exact magnetic field of the magnet at any point in space has some mathematical complexities and involves the multiple properties of the magnetic materials. Things are simpler using direct measures of the magnetic field discussed in the previous section, such as the equation obtained for the horizontal component of the magnetic field $B_{x}$ of the magnet in the monopolar configuration, as discussed in $[4,5]$, which is given by

$$
B_{x}=\frac{D\left(1-x^{2}\right)}{4 \pi\left(x^{2}+D^{2}\right)^{5 / 2}}
$$

where $D$ is a constant featured for this magnet and $x$ is the distance.

The formation of the needlelike structures of Figure 1(a) is directly related to the magnetic field $B$ around $200 \mathrm{G}$; the direction of the polarization of the needles inside the circular region, with radius $R$ and width $a$, is given by the coordinates $\left(x_{i}, y_{i}\right)$ and $\left(x_{i+1}, y_{i+1}\right)$ for the angle $\theta$ :

$$
\left(\begin{array}{ll}
x_{i} & x_{i+1} \\
y_{i} & y_{i+1}
\end{array}\right)=\left(\begin{array}{c}
\cos \theta \\
\sin \theta
\end{array}\right)(R(R+a)) .
$$

Using (1) and (2), we are proposing that the light intensity of the pattern observed in the Ferrocell for the monopolar configuration $[4,5]$ is given by

$$
I(r, \theta)=\frac{D\left(1-r^{2}\right)}{16 \pi\left(r^{2}+D^{2}\right)^{5 / 2}} \sin ^{2} 2 \theta,
$$

where $r$ ranges from 0 to infinity and $\theta$ ranges from $0^{\circ}$ to $360^{\circ}$.
This pattern suggested empirically a resemblance of this system with the patterns involving the concept of spatial modes of electromagnetic radiation in an open-sided resonator. In order to explore some of the interesting patterns observed with the traditional solutions of paraxial wave equation [6], we have used a simple hint used in computational graphics: the pattern recognition. Based on the idea that any picture may be conceived as a mathematical object, lying on part of the Euclidean plane and each point having its own light intensity, we have explored the pattern formation using matrices with $1000 \times 1000$ elements, developing and testing several mathematical functions. Even though this phenomenological method is very empirical and takes a lot of time to find some useful images, many commercial software packages available have a wealth of built-in mathematical functions, facilitating the exploration of image formation.

Following the previous method, we have found that the mathematical problem of image formation involves as solutions for this kind of system the Hermite-Gauss and other hypergeometric polynomials [7]. We have approximated the intensity of the light polarization observed for the magnetic field generated by these magnets by

$$
\begin{aligned}
& B(x, y, z)=B_{0} H_{m}(x) H_{n}(y) e^{-\left(x^{2}+y^{2}\right) / w^{2}(z)} e^{i \phi(z)}, \\
& I(x, y, z)=|B(x, y, z)|^{2},
\end{aligned}
$$

in which $B_{0}$ is the maximal intensity of the magnetic field and $H_{m}(x)$ and $H_{n}(y)$ are the spatial derivatives of the functions of the magnetic field in the $x$-axis and $y$-axis. $I(x, y, z)$ is the light intensity observed. We also have used the expressions of the magnetic field in non-Cartesian coordinates in order to obtain different types of light patterns, besides HermiteGauss polynomials.

Changing the orientation of the magnetic field, we have obtained different configuration patterns. For example, in Figure 5(a), we present some patterns obtained for the case of the bipolar configuration. The case of the compound magnets 


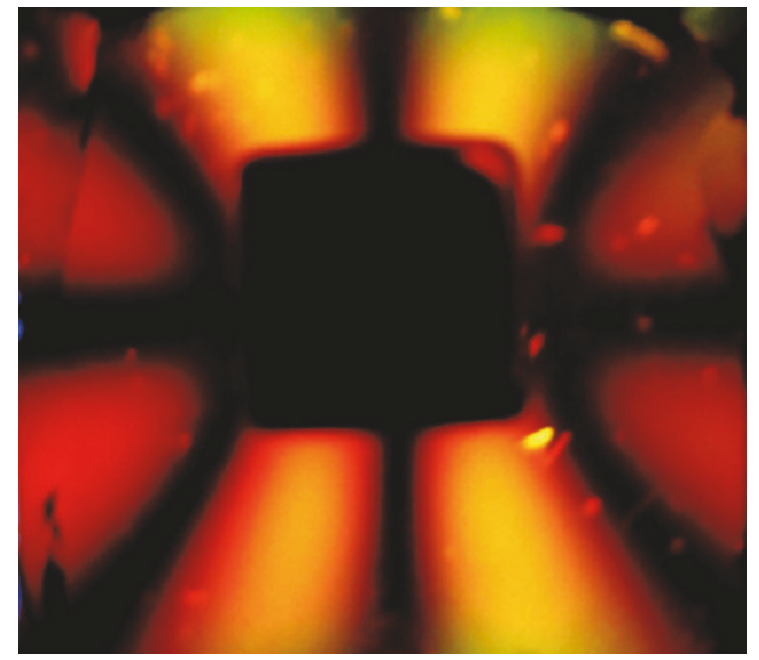

(a)

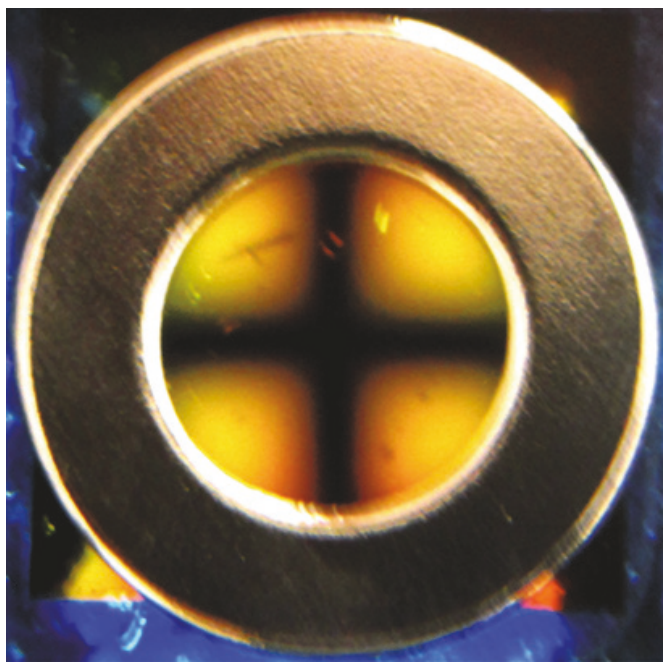

(c)

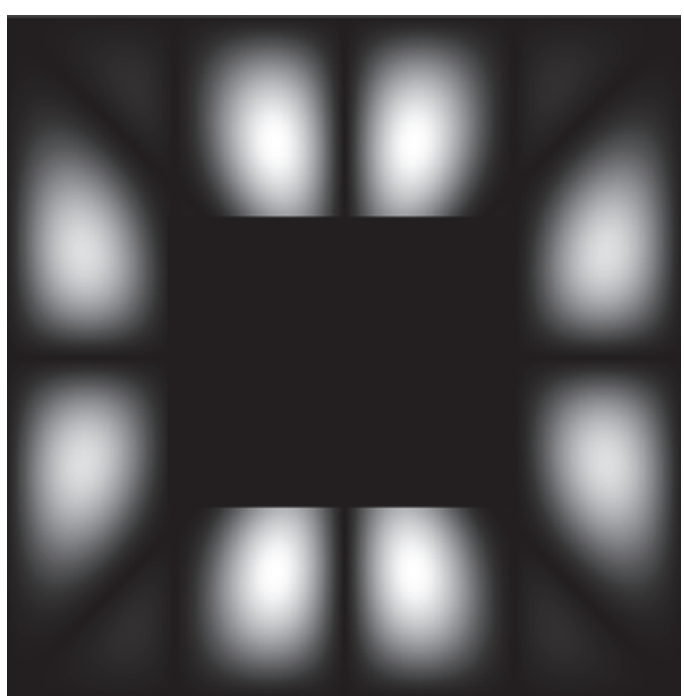

(e)

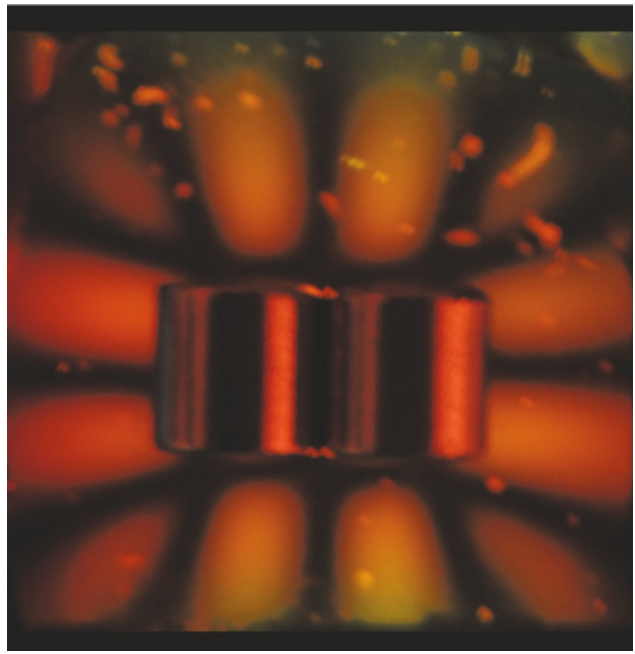

(b)

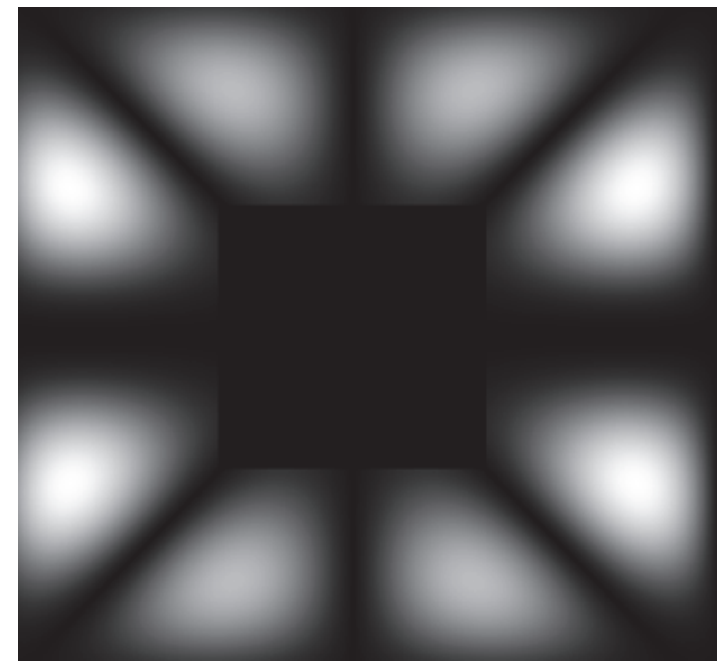

(d)

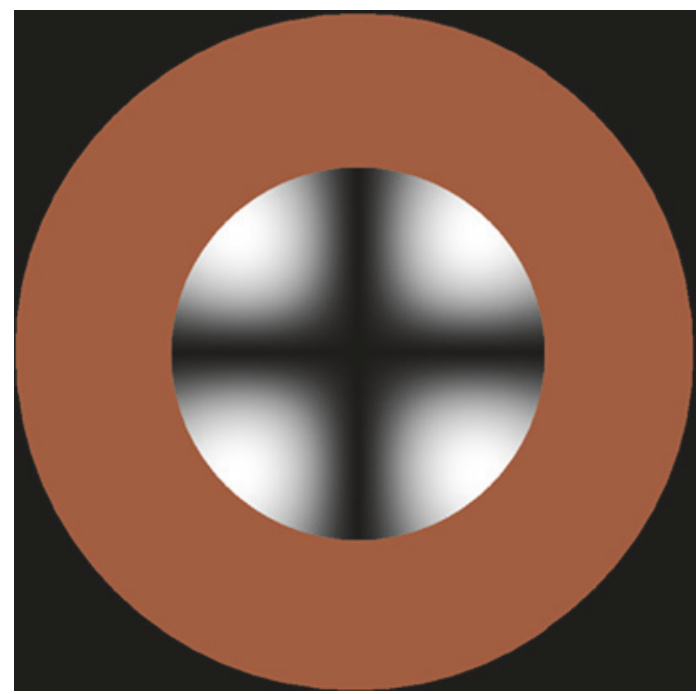

(f)

Figure 5: Patterns obtained with bipolar configuration (a); (b) tetrapolar configuration; (c) using a magnetic ring. Simulations for bipolar configuration (d); (e) tetrapolar configuration; (f) for the case of the magnetic ring. 
(a)

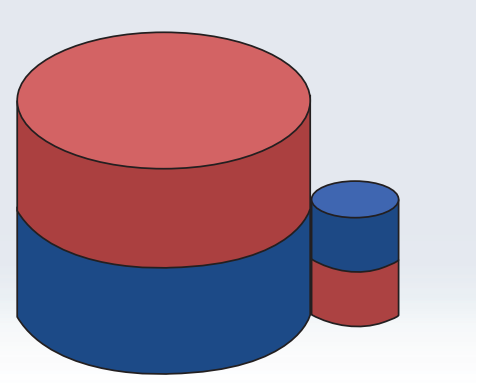

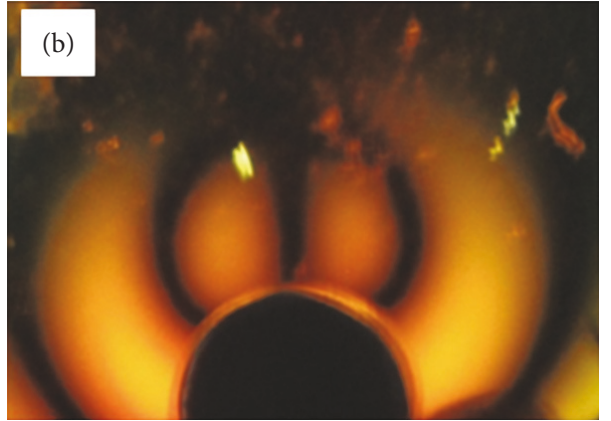

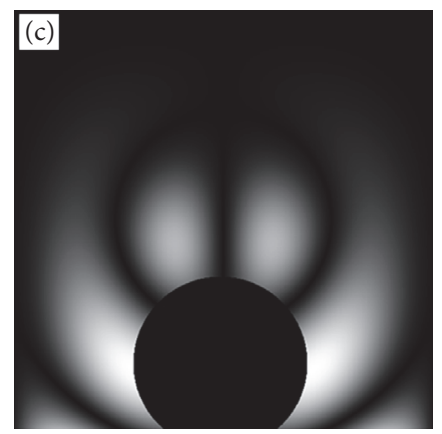

Figure 6: The magnetic field of two cylindrical magnets, placed side by side with poles in opposition, as it is shown in the diagram (a), one magnet with 900 Gauss and the small one with 200 Gauss. The pattern obtained with the Ferrocell is shown in (b) with the small magnet at the bottom. (c) presents the simulation for this compound magnet.

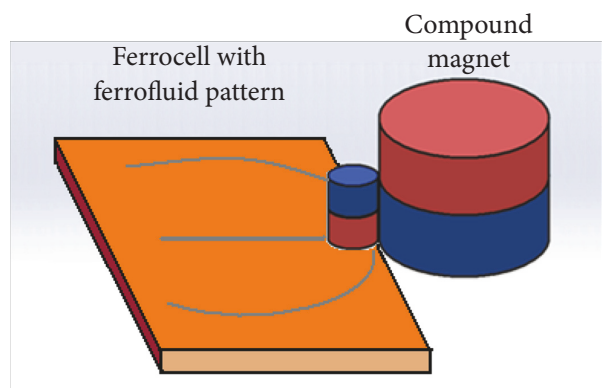

(a)

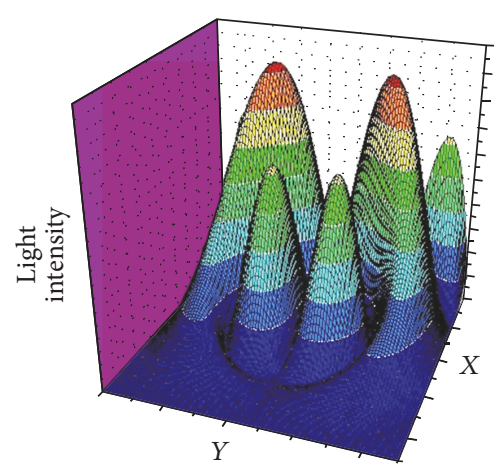

(b)

FIGURE 7: The diagram of the magnets of the previous case in (a) and the plot of the light intensity of two cylindrical magnets with different sizes and magnetic field intensities in (b), showing the result of the magnetic field of the small magnet being embraced by the magnetic field of the large magnet, resembling the interaction in the solar wind being deflected by the magnetic field of the earth.

in the tetrapolar configuration is shown in Figure 5(b), and this pattern is associated with the magnetic field of Figure $3(\mathrm{~d})$. The pattern of light polarization of a magnetic ring is shown in Figure 5(c). The respective simulation of each case is presented in Figure 5(d) for bipolar configuration, in Figure 5(e) for the tetrapolar configuration, and in Figure 5(f) for the case of the magnetic ring.

These patterns indicate that the Ferrocell has the same role of a diffraction grating in the presence of the magnetic field, similar to the case of liquid crystal devices $[1,2]$.

An interesting case to report is the light pattern obtained for a compound magnet of Figure 6, with two cylindrical magnets placed side by side with poles in opposition, in which a more intense magnetic field "embraces" the magnetic field of the smaller cylindrical magnet. Due to the symmetry of this system, this pattern resembles one of the modes of the Laguerre-Gauss polynomials, with decay of the magnetic field pointing to the top of Figure 6(c). The diagram of this setup is represented in Figure 7(a), and the light intensity of this configuration is shown in Figure 7(b), without the representation of the small magnet.

One possible application of this system is with physics demonstrations, in order to introduce the students to more complex concepts observed in nature, because the reaction of the ferrofluid to the external magnetic field is very fast. For example, this setup can be used to construct demonstrations of the interaction between the extreme solar wind conditions stressing the earth magnetosphere, moving one of the magnets, while the small magnet is kept fixed.

As a final note, in order to show the complex behavior of the Ferrocell in the presence of magnetic field of a ring, we present in Figure 8 a different setup just using a round Ferrocell and two lasers crossing each other, in the same plane of the Ferrocell. There are no polarizers in this setup, and the magnetic ring is covered with an opaque sheet of paper, so the image formed is only due to the arrangement of the ferrofluid particles scattering the light. Although this setup is out of the scope of the present study, many important features of the Ferrocell used in the polarization study can be found in [8], in which the authors describe some techniques for the visualization of magnetic fields and explain in more detail the constitutive aspects of the Ferrocell.

\section{Conclusions}

We have presented an experimental setup with potential application in characterizing and controlling the quality of magnets, enabling the visualization and a fast analysis of the 


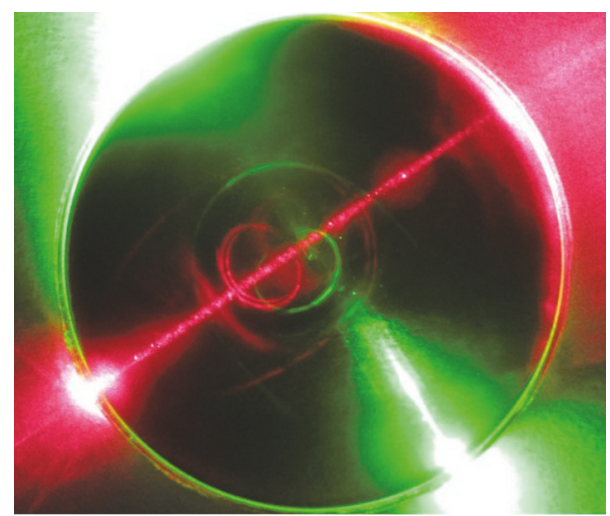

FIgURE 8: Two crossed laser beams (red and green) in a circular Ferrocell with a magnetic ring behind it. There is no polarizer in this setup. We can observe the ferrofluid particles scattering light forming curved structures at the center of the picture, which resembles some parts of the magnetic ring.

magnetic field of small multipolar magnets and magnetic assemblies. The orientation of the particles inside the ferrofluid controls the transmittance intensity.

The pattern formation curiously resembles the nodal lines of the transverse electric and magnetic modes observed in optical resonant cavities, associated with the hypergeometric polynomials, such as Hermite-Gauss, Laguerre-Gauss, and Ince-Gauss polynomials. In this analogy, the magnetic field has an equivalent role of the Gaussian irradiance distribution, and the light source is a very flat lamp normal to $z$, which nearly corresponds to a plane wave with constant flux set up to the $z$-axis, analogous to a standing wave in the optical cavity.

\section{Conflicts of Interest}

The authors declare that they have no conflicts of interest.

\section{Acknowledgments}

This work was partially supported by Conselho Nacional de Desenvolvimento Científico e Tecnológico (CNPq), Instituto Nacional de Ciência e Tecnologia de Fluidos Complexos (INCT-FCx), and by Fundação de Amparo à Pesquisa do Estado de São Paulo (FAPESP), FAPES/CNPq\#573560/20080 .

\section{References}

[1] Y.-H. Wu, Y.-H. Lin, H. Ren, X. Nie, J.-H. Lee, and S.-T. Wu, "Axially-symmetric sheared polymer network liquid crystals," Optics Express, vol. 13, no. 12, pp. 4638-4644, 2005.

[2] S. Nersisyan, N. Tabiryan, D. M. Steeves, and B. R. Kimball, "Fabrication of liquid crystal polymer axial waveplates for UVIR wavelengths," Optics Express, vol. 17, no. 14, pp. 11926-11934, 2009.

[3] C. Penfold, R. T. Collins, A. P. B. Tufaile, and Y. Souche, "Transverse magneto-optical Kerr effect: the phase change of reflected light," Journal of Magnetism and Magnetic Materials, vol. 242-245, pp. 964-966, 2002.

[4] A. Tufaile, T. A. Vanderelli, and A. P. B. Tufaile, "Light polarization using ferrofluids and magnetic fields," in Encontro de Física 2016, Natal, Brazil, September 2016.

[5] A. Tufaile, T. A. Vanderelli, and A. P. Biscaia Tufaile, "Observing the jumping laser dogs," Journal of Applied Mathematics and Physics, vol. 04, no. 11, pp. 1977-1988, 2016.

[6] G. R. Fowles, Introduction to Modern Optics, Dover, New York, NY, USA, 2013.

[7] M. A. Bandres and J. C. Gutiérrez-Vega, "Ince-Gaussian modes of the paraxial wave equation and stable resonators," Journal of the Optical Society of America A, vol. 21, no. 5, pp. 873-880, 2004.

[8] M. Snyder and J. Frederick, "Photonic dipole contours of ferrofluid Hele-Shaw cell," https://arxiv.org/ftp/arxiv/papers/0805/ 0805.4364.pdf. 

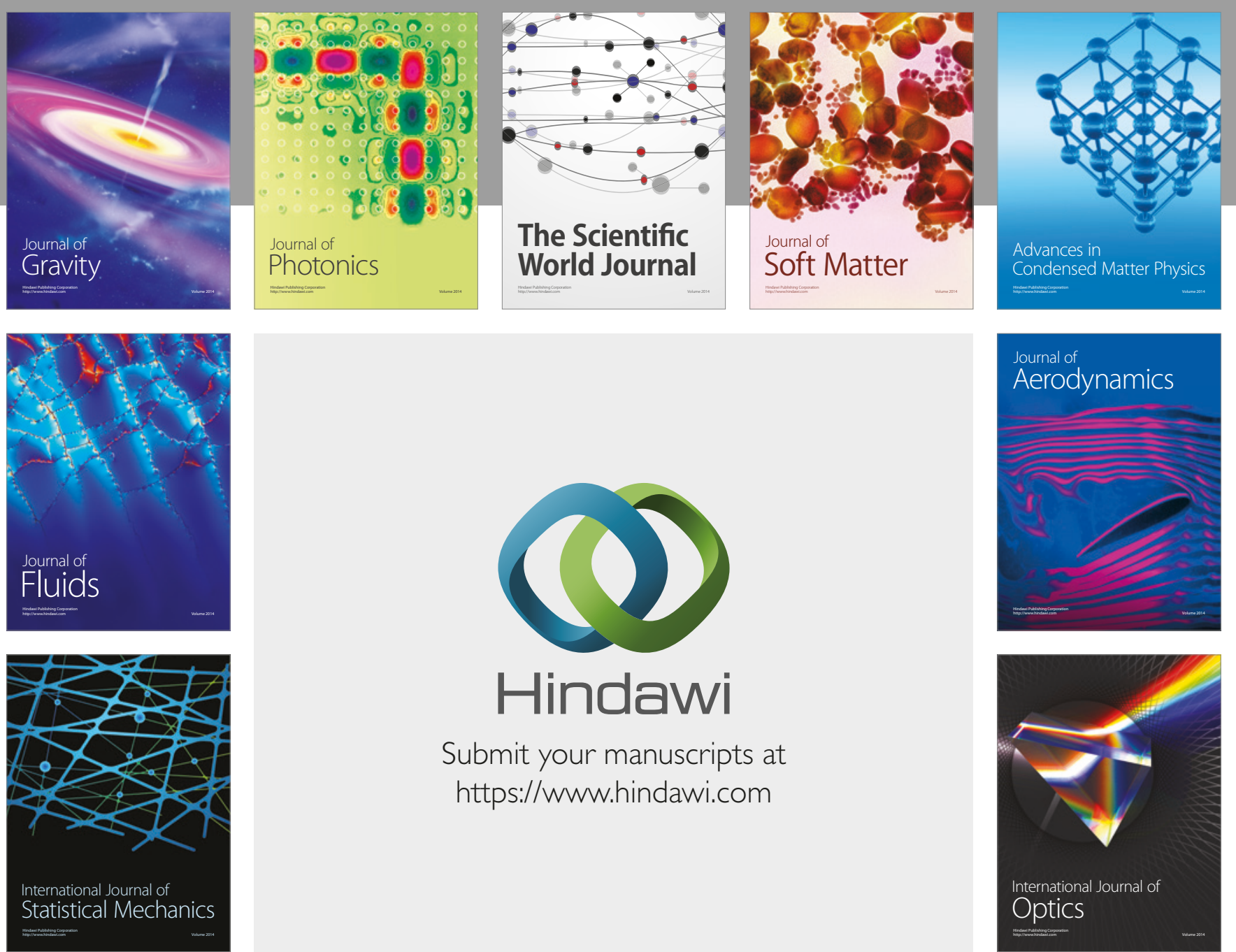

Submit your manuscripts at

https://www.hindawi.com
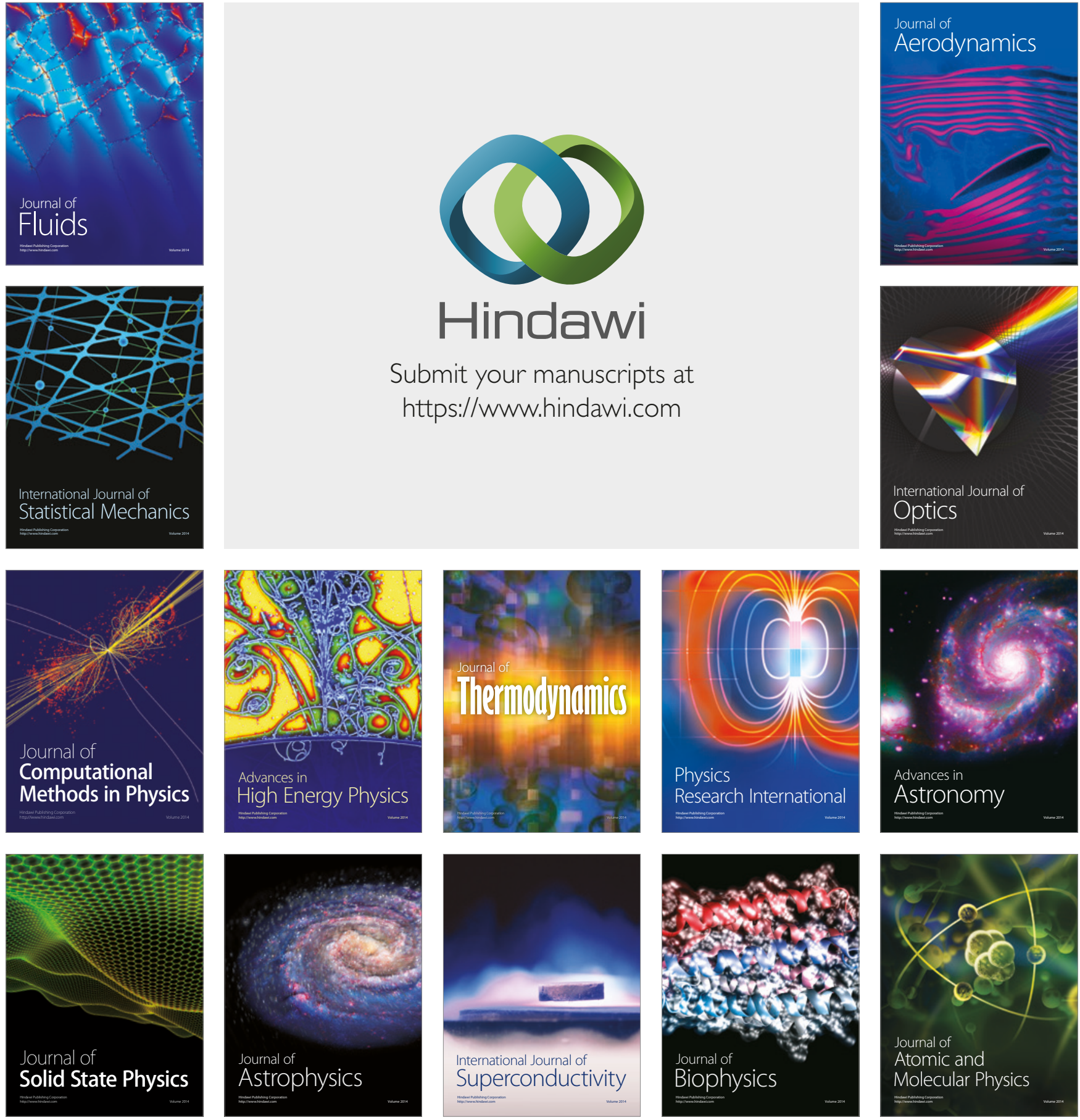